\title{
REDUÇÃO DO AMIDO DIETÉTICO, UTILIZANDO ÓLEO DE SOJA, EM DIETAS PARA COELHOS EM CRESCIMENTO
}

\author{
Dietary starch reduction, using soybean oil, in diets for growing rabbits
}

\author{
Jeffrey Frederico Lui', Estácio Alves dos Santos ${ }^{2}$, Antonio Carlos Homem Junior ${ }^{3}$, Maria Cristina de Oliveira ${ }^{4}$, \\ Luciana Cardoso Cancherini ${ }^{5}$
}

\begin{abstract}
RESUMO
Este experimento foi conduzido para avaliar os efeitos da redução do amido dietético, utilizando óleo de soja, em dietas para coelhos Nova Zelândia Branco em crescimento sobre o desempenho e rendimento de carcaça dos animais. Os coelhos foram desmamados aos 30 dias e abatidos aos 60 dias de idade. O delineamento experimental foi em blocos ao acaso com quatro tratamentos $(2,2,3,3,4,4$ e 5,5\% de adição de óleo de soja às dietas) e cinco repetições com 15 animais por tratamento. Foram avaliados os ganhos de peso diário, a ingestão de alimentos diária, a conversão alimentar, a mortalidade e o rendimento de carcaça. Não houve efeito $(P>0,05)$ dos tratamentos sobre os parâmetros avaliados. Concluiu-se que, nas condições realizadas neste trabalho, a adição de óleo de soja em dietas para coelhos em crescimento até o nível de 5,5\% não afetou o desempenho produtivo.
\end{abstract}

Termos para indexação: Desempenho, fontes de energia, rendimento de carcaça.

\begin{abstract}
This experiment was carried out to evaluate the effects of dietary starch reduction, in diets for growing White New Zealand rabbits, using soybean oil, on the performance and carcass yield of the animals. The rabbits were weaned at 30 days and slaughtered at 60 days of age. The experimental design was in randomized blocks with four treatments $(2.2,3.3,4.4$ and $5.5 \%$ of soybean oil addition to the diets) and five replicates, with 15 animals per treatment. Daily weight gain, daily feed intake, feed conversion rate, mortality and carcass yield were evaluated. There was no effect $(\mathrm{P}>0.05)$ due the treatments on the evaluated parameters. It was concluded that, at the conditions of this work, the addition of soybean oil in growing rabbit diets up to the level of 5.5\% did not affect the productive performance.
\end{abstract}

Index terms: Carcass yield, energy sources, performance.

(Recebido para publicação em 18 de janeiro de 2005 e aprovado em 3 de julho de 2006)

\section{INTRODUÇÃO}

O amido é uma das principais fontes de energia nas dietas para coelhos. No entanto, níveis elevados deste nutriente podem causar disfunções digestivas, tais como: diarréias, alterações no padrão de fermentação cecal (FARIA et al., 2004), modificações na produção dos ácidos graxos voláteis, desidratação por mudanças na pressão osmótica intestinal, disbioses microbianas e altas taxas de mortalidade na fase de crescimento (ARRUDA et al., 2003). Quando o nível de amido é alto e de fibra indigestível é baixo nas dietas corre-se o risco de haver alterações também na microflora intestinal, no processo de cecotrofia e no rendimento produtivo do animal (CHEEKE, 1995).

Lipídios exercem funções essenciais no organismo animal sendo fonte de energia, precursores de componentes biológicos (prostaglandinas, hormônios esteróides, sais biliares, ácidos graxos essenciais etc), mantendo a integridade das membranas celulares e lipoproteínas e estando relacionados à redução do pó e aumento da palatabilidade da dieta (MAERTENS et al., 1998).

A concentração energética é uma das principais variáveis na formulação de dietas para coelhos em crescimento. Os coelhos podem crescer bem em uma ampla faixa de níveis de fibras dietéticas (DE-BLAS et al., 1986). A suplementação das dietas com gorduras permite um aumento no nível de fibra ou na concentração de energia das dietas pobres em fibras (BHATT \& SWAIN, 2003).

Assim como em outros monogástricos, o controle da ingestão de alimentos pelos coelhos se dá pelo nível energético das dietas. Segundo Cheeke (1987), o ajuste fisiológico do consumo se dá em uma amplitude de 2200 a $3200 \mathrm{kcal} \mathrm{ED} / \mathrm{kg}$ de dieta. O teor de energia bruta dos óleos é quase três vezes maior do que de outros alimentos e, assim sendo, altos níveis de óleo são usados para produzir

\footnotetext{
'Professor do Departamento de Zootencia - FCAV/UNESP - Rua Donato Castellane, s/n - 14.880-900 - Jaboticabal, SP - jeffrey@fcav.unesp.br ${ }^{2}$ Professor do Departamento de Zootecnia - Universidade Estadual do Piauí - Rua Joaquim Nogueira de Oliveira s/n - 64.980-000 - Corrente, PI tarso99@ig.com.br

${ }^{3}$ Estudante de Zootecnia - FCAV/UNESP - Rua Donato Castellane, s/n - 14.880-900 - Jaboticabal, SP.

${ }^{4}$ Professora do Departamento de Zootecnia - Universidade de Rio Verde - Fazenda Fontes do Saber s/n - 75.901-970 - Rio Verde, GO - cristina@fesurv.br ${ }^{5}$ Professora do Departamento de Zootecnia - Universidade do Estado de Mato Grosso - Av. Tancredo Neves, 1095 - $78.200-000$ - Cáceres, MT cll282@hotmail.com
} 
dietas ricas em energia. Além disso, a energia representa altos custos na formulação de dietas e os óleos são bem competitivos neste sentido.

Vários autores mencionaram que a adição de óleo às dietas pode melhorar o peso corporal (ARRIGTON et al., 1974), a conversão alimentar (FERNÁNDEZ \& FRAGA, 1996), o ganho de peso (EL-HUSSEINY et al., 2004), reduzir o consumo de alimentos (CUNHA et al., 1998) e diminuir a mortalidade (SOLER et al., 2004). Enquanto outros (CARREGAL \& MURAKAMI, 1980) relataram não haver efeitos sobre o desempenho dos coelhos.

Este experimento foi desenvolvido para estudar a substituição do amido por óleo vegetal em dietas para coelhos em crescimento.

\section{MATERIAL E MÉTODOS}

O experimento foi conduzido no Setor de
Cunicultura da FCAV/UNESP no período de maio a julho de 2002. A temperatura e a umidade relativa do ar no período foram $20,92^{\circ} \mathrm{C}$ e $57 \%$, respectivamente. Foram utilizados 60 coelhos machos da raça Nova Zelândia Branco, com peso inicial médio de $754 \pm 48,09 \mathrm{~g}$, desmamados aos 30 dias e alojados em gaiolas de arame galvanizado ( $40 \times 60 \times 45 \mathrm{~cm}$ ), equipadas com comedouro semi-automático e bebedouro tipo chupeta. Os animais receberam ração e água à vontade durante os 30 dias de duração do experimento.

O delineamento experimental foi em blocos casualizados repetidos no tempo, com quatro tratamentos e cinco repetições de três animais por gaiola. Os tratamentos consistiram de níveis de inclusão de óleo de soja em dietas (Tabela 1) formuladas para atender as recomendações para coelhos em crescimento (INRA, 1999). Os níveis de óleo utilizados foram 2,2, 3,3, 4,4 e 5,5\%.

TABELA 1 - Composição percentual das rações experimentais.

\begin{tabular}{|c|c|c|c|c|}
\hline \multirow[t]{2}{*}{ Ingredientes (kg) } & \multicolumn{4}{|c|}{ Nível de inclusão de óleo de soja (\%) } \\
\hline & 2,2 & $\mathbf{3 , 3}$ & 4,4 & 5,5 \\
\hline Milho moído & 15,20 & 15,20 & 15,20 & 15,20 \\
\hline Farelo de soja & 19,60 & 19,60 & 19,60 & 19,60 \\
\hline Farelo de trigo & 13,20 & 13,20 & 13,20 & 13,20 \\
\hline Feno de tifton & 36,00 & 36,00 & 36,00 & 36,00 \\
\hline Casca de arroz & 1,40 & 1,40 & 1,40 & 1,40 \\
\hline Óleo de soja & 2,20 & 3,30 & 4,40 & 5,50 \\
\hline Amido & 8,00 & 5,60 & 3,20 & - \\
\hline Sal & 0,50 & 0,50 & 0,50 & 0,50 \\
\hline Calcário calcítico & 0,80 & 0,80 & 0,80 & 0,80 \\
\hline Fosfato bicálcico & 0,30 & 0,30 & 0,30 & 0,30 \\
\hline Suplemento mineral e vitamínico ${ }^{1}$ & 0,30 & 0,30 & 0,30 & 0,30 \\
\hline Coccidiostático & 0,08 & 0,08 & 0,08 & 0,08 \\
\hline Caulim & 2,42 & 3,72 & 5,02 & 7,12 \\
\hline \multirow[t]{2}{*}{ Total } & 100,00 & 100,00 & 100,00 & 100,00 \\
\hline & \multicolumn{4}{|c|}{ Composição calculada ${ }^{2}$} \\
\hline Matéria seca (\%) & 85,12 & 84,08 & 83,04 & 81,29 \\
\hline Proteína bruta $(\%)$ & 16,40 & 16,40 & 16,40 & 16,40 \\
\hline Energia digestível $(\mathrm{kcal} / \mathrm{kg})^{3}$ & 2535 & 2541 & 2547 & 2524 \\
\hline Fibra em detergente ácido (\%) & 21,52 & 21,52 & 21,52 & 21,52 \\
\hline Fibra em detergente neutro (\%) & 39,04 & 39,04 & 39,04 & 39,04 \\
\hline Extrato etéreo $(\%)$ & 3,55 & 4,64 & 5,72 & 6,80 \\
\hline Amido $(\%)$ & 23,79 & 21,68 & 19,57 & 16,76 \\
\hline Cálcio (\%) & 0,51 & 0,51 & 0,51 & 0,51 \\
\hline Fósforo (\%) & 0,31 & 0,31 & 0,31 & 0,31 \\
\hline
\end{tabular}

${ }^{1}$ composição por kg do produto: Vit A, 1.875 .000 UI; Vit $D_{3}, 250.000$ UI: Vit E, 12.500 mg; Vit K, 750 mg; Vit B, 500 mg; Vit B, $1.000 \mathrm{mg}$; Vit B , $600 \mathrm{mg}$; Vit B, $2.500 \mathrm{mcg}$; Ácido Pantotênico, $2000 \mathrm{mg}$; Colina, $125 \mathrm{~g}$; Fe, $80.000 \mathrm{mg}$; Cu, 12.000 mg; Mn, 60.000 mg; Zn, 50.000 mg; I, 5.000 mg; Se, 15 mg; Antioxidante, 25 g.

${ }^{2}$ Segundo INRA (1999) e Rostagno et al. (2000), baseado na matéria natural.

${ }^{3}$ Valor de energia digestível do feno de tifton segundo NRC (1977). 
Os animais e as dietas foram pesados no início e no final do experimento para obtenção do ganho de peso e do consumo de ração diários e da conversão alimentar. A mortalidade também foi determinada. Quando os animais atingiram o peso médio de $1886 \pm 56 \mathrm{~g}$, aos 60 dias de idade, eles foram submetidos a jejum alimentar de 12 horas e, após esse período, foram novamente pesados para obtenção do peso ao abate. As carcaças evisceradas, sem cabeça e pés, foram pesadas e seus rendimentos foram calculados em função do peso ao abate.

A análise estatística dos resultados foi feita por meio de análise de regressão polinomial do programa SAEG (UFV, 1997) e para a comparação entre as médias utilizouse o teste Tukey.

\section{RESULTADOS E DISCUSSÃO}

Não houve influência dos tratamentos $(\mathrm{P}>0,05)$ sobre o ganho de peso diário, ingestão de alimento diária, conversão alimentar, mortalidade e rendimento de carcaça (Tabela 2).

$\mathrm{Na}$ medida em que os níveis de amido foram reduzidos o teor de matéria seca das dietas também diminuiu (Tabela 1). Como o consumo de ração não diferiu entre os tratamentos, pode-se inferir que os coelhos alimentados com dietas contendo menores níveis de amido consumiram quantidades menores de matéria seca. Como o óleo tem a capacidade de tornar as dietas mais palatáveis, era esperado um aumento no consumo de ração em função dos níveis de óleo na dieta que não ocorreu devido, provavelmente, ao fato de que as dietas eram isocalóricas e os coelhos controlam sua ingestão de alimentos pelo nível energético das dietas. Bhatt \& Swain (2003) também não notaram efeito no consumo de alimentos e no rendimento de carcaça ao estudarem dietas com níveis de inclusão de 2 a $8 \%$ de óleo vegetal, porém, Fernández \& Fraga (1996) que utilizaram dietas com 3\% de óleo de soja e Cunha et al. (1998) que avaliaram a inclusão de 0, 3,5 e 7\% de gorduras em dietas para coelhos, observaram que o consumo de ração diminuiu com a inclusão do óleo.

O menor consumo de matéria seca não interferiu nos resultados de ganho de peso devido, provavelmente, ao consumo de energia dietética que não diferiu entre os tratamentos. Se a energia das dietas tivesse aumentado com o aumento da inclusão de óleo, os animais poderiam ter seu ganho de peso aumentado também, pois segundo Bhatt \& Swain (2003) a maior concentração energética promove maior utilização de proteínas e gorduras das dietas.

Ausência de efeito da inclusão de óleo em dietas para coelhos sobre o ganho de peso foi relatada por Fernández \& Fraga (1996) (3\% de óleo de soja). Bhatt \& Swain (2003), entretanto, ao trabalharem com 0, 2, 4, 6 e $8 \%$ de óleo vegetal na dieta, observaram maiores ganhos de peso com inclusão de 6 e $8 \%$ e El-Husseiny et al. (2004) (3, 6 e 9\% de óleo) relataram que o ganho de peso foi melhor com o nível de $9 \%$.

Como o consumo e o ganho de peso foram semelhantes em todos os tratamentos, a conversão alimentar não foi afetada. Melhor conversão alimentar devido à adição de óleo em dietas para coelhos foi mencionado por Fernández \& Fraga (1996) que verificaram melhor conversão alimentar com a inclusão de 3\% de óleo. Já Cunha et al. (1998) notaram piora na conversão alimentar de coelhos ingerindo dietas contendo 4 e $8 \%$ de óleo $(3,36$ e 4,24, respectivamente) comparado com a dieta controle (3,29). Da mesma forma, Arruda et al. (2000) relataram que coelhos alimentados com dietas contendo o maior nível de óleo de soja $(8,13 \%)$ apresentaram a pior conversão alimentar (4,02). El-Husseiny et al. (2004) analisaram três níveis de óleo (3, 6 e 9\%) na dieta de coelhos e relataram que a conversão alimentar foi melhor com a inclusão de 3 e $6 \%$ de óleo.

TABELA 2 - Desempenho e rendimento de carcaça de coelhos recebendo dietas com substituição do amido por óleo de soja.

\begin{tabular}{lccccc}
\hline Parâmetros & \multicolumn{3}{c}{ Níveis de inclusão de óleo de soja (\%) } & \multirow{2}{*}{ EPM $^{\mathbf{1}}$} \\
\cline { 2 - 5 } & $\mathbf{2 , 2}$ & $\mathbf{3 , 3}$ & $\mathbf{4 , 4}$ & $\mathbf{5 , 5}$ & \\
\hline Ganho de peso médio diário (g/d) & 38,97 & 36,50 & 38,40 & 37,03 & 0,37 \\
Consumo de ração médio diário (g/d) & 122,80 & 123,04 & 119,92 & 122,94 & 0,48 \\
Conversão alimentar & 3,15 & 3,37 & 3,12 & 3,32 & 0,03 \\
Mortalidade (\%) & 0 & 0 & 0 & 0 & - \\
Rendimento de carcaça (\%) & 51,80 & 52,00 & 51,60 & 51,60 & 0,25 \\
\hline
\end{tabular}

1 - Erro padrão da média. 


\section{CONCLUSÃO}

O óleo de soja pode ser incluído em até 5,5\% em substituição ao amido na dieta de coelhos em crescimento.

\section{REFERÊNCIAS BIBLIOGRÁFICAS}

ARRINGTON, L. R.; PLATT, J. K.; FRANKE, D. E. Fat utilization by rabbits. Journal of Animal Science, Champaign, v. 38, n. 1, p. 76-80, 1974.

ARRUDA, A. M. V.; CARREGAL, R. D.; FERREIRA, R. G. Desempenho produtivo e atividade microbiana cecal de coelhos alimentados com dietas contendo diferentes níveis de amido. Revista Brasileira de Zootecnia, Viçosa, v. 29, n. 3, p. 762-768, 2000.

ARRUDA, A. M. V.; LOPES, D. C.; FERREIRA, W. M.; ROSTAGNO, H. S.; QUEIROZ, A. C.; PEREIRA, E. S.; SILVA, J. F.; JHAM, G. N. Atividade microbiana cecal e contribuição nutricional da cecotrofia em coelhos alimentados com rações contendo diferentes fontes de fibra e níveis de amido. Revista Brasileira de Zootecnia, Viçosa, v. 32, n. 4, p. 891-902, 2003.

BHATT, R. S.; SWAIN, N. Effect of graded level of fat supplementation on the growth performance in the rabbits. World Rabbit Science, [S.l.], v. 11, n. 1, p. 33-40, 2003.

CARREGAL, R. D.; MURAKAMI, A. E. Efeito da adição de níveis de óleo vegetal em rações de coelhos em crescimento. Revista Latino-Americana de Cunicultura, [S.1.], v. 1, p. 17-20, 1980.

CHEEKE, P. R. Alimentación y nutrición del conejo. Zaragoza: Acribia, 1995. 429 p.

CHEEKE, P. R. Rabbit feeding and nutrition. Oregon: Academic, 1987. 380 p.

CUNHA, L. F. e; FERREIRA, P.; FREIRE, J. P. B. Fiber x lipid interaction in rabbit diets: growth, digestibility and fermentation patterns. World Rabbit Science, [S.1.], v. 6, p. 22, 1998.

DE-BLAS, J. C.; SANTOMA, G.; CARABAÑO, R.; FRAGA, M. J. Fiber and starch levels in fattening rabbit diets. Journal of Animal Science, Champaign, v. 63, n. 3, p. 1897-1904, 1986.
EL-HUSSEINY, O.; GHAZALAH, A. A.; ARAFA, S. A.; EL-MANYLAWI, M. A. Response of growing rabbit performance to some dietary vegetable oils and their Casoap. World Rabbit Science, [S.1.], v. 12, n. 3, p. 214, 2004.

FARIA, H. G.; SCAPINELLO, C.; PERALTA, R. M.; GIDENNE, T.; FURLAN, A. C.; ANDREAZZI, M. A. Digestibilidade e desempenho de coelhos oriundos de quatro padrões de alimentação até a desmama alimentados com dietas contendo diferentes níveis de amido após a desmama. Revista Brasileira de Zootecnia, Viçosa, v. 33, n. 5, p. 1172-1180, 2004.

FERNÁNDEZ, C.; FRAGA, M. J. Effect of fat inclusion in diets for rabbits on the efficiency of digestible energy and protein utilization. World Rabbit Science, [S.l.], v. 4, n. 1, p. 19-23, 1996

INSTITUT NATIONAL DE LA RECHERCHE AGRONOMIQUE. Alimentação dos animais monogástricos: suínos, coelhos e aves. 2. ed. São Paulo: Roca, 1999. 245 p.

MAERTENS, L.; CAVANI, C.; LUZI, F.; CAPOZZI, F. Influence du rapport protéines/énergie et de la source ènergétique de $1^{\prime}$ 'aliment sur les performances, l'excrétion azotée et les caractéristiques de la viande des lapins em finition. In: JOURNÉES DE LA RESEARCHE CUNICULE, 7., 1998, Lyon. Proceedings... Lyon: WRSA, 1998. p. 163166.

NATIONAL RESEARCH COUNCIL. Nutrient requirement of rabbits. Washington, DC: National Academy of Science, 1977.

ROSTAGNO, H. S.; ALBINO, L. F. T.; DONZELE, J. L.; GOMES, P. C.; FERREIRA, A. S.; OLIVEIRA, R. F.; LOPES, D. C. Tabelas brasileiras para aves e suínos: composição de alimentos e exigências nutricionais. Viçosa: UFV, 2000. 141 p.

SOLER, M. D.; BLAS, E.; CANO, J. L.; PASCUAL, J. J.; CERVERA, C.; FERNÁNDEZ-CARMONA, J. Effect of the relationship between digestible fibre/starch and the fat content of starter feed on the mortality rate of rabbits. World Rabbit Science, [S.1.], v. 12, n. 3, p. 129, 2004.

UNIVERSIDADE FEDERAL DE VIÇOSA. Manual de utilização do programa SAEG - Sistema para análise estatísticas e genéticas. Viçosa, 1997.59 p. 